\title{
A non-local fracture model for composite laminates and numerical simulations by using the FFT method
}

\author{
Jia Li $^{\mathrm{a}, *}$, Songhe Meng ${ }^{\mathrm{b}}$, Xiaoxiao Tian ${ }^{\mathrm{a}, \mathrm{b}}$, Fan Song ${ }^{\mathrm{c}}$, Chiping Jiang ${ }^{\mathrm{d}}$ \\ a LSPM, CNRS UPR 3407, Université Paris XIII, Villetaneuse, France \\ ${ }^{\mathrm{b}}$ Center for Composite Materials, Harbin Institute of Technology, Harbin, China \\ ' State Key Laboratory of Nonlinear Mechanics, Institute of Mechanics, Chinese Academy of Sciences, Beijing, China \\ ${ }^{\mathrm{d}}$ Solid Mechanics Research Center, Beijing University of Aeronautics and Astronautics, Beijing, China
}

\section{A R T I C L E I N F O}

\section{Article history:}

Received 20 July 2011

Received in revised form 23 August 2011

Accepted 31 August 2011

Available online 17 September 2011

\section{Keywords:}

A. Laminate

B. Fracture

B. Interface/interphase

C. Damage mechanics

\begin{abstract}
A B S T R A C T
In this paper, we present a fracture model for composite laminates and its numerical solution by using the Fast Fourier Transforms (FFTs). The FFT-based formulation initially proposed for seeking the average behaviour of linear and non-linear composites by means of the homogenisation procedures [1,2] was adapted to evaluate the damage growth in brittle materials. A non-local damage model based on the maximal principal stress criterion was proposed to assess the failure in the matrix and the fibres. This non-local model was then connected to the Griffith-Irwin criterion in the aim of predicting crack growth. In order to assess the matrix/fibre interface delamination, we have adapted the cohesive model developed by Li [3] for accounting the mixed-mode dependent interface failure. To this end, the interfaces between the matrix and the fibres are replaced by a thin layer of interphase with the purpose of facilitating the FFT simulations. By using the proposed model, we carried out several numerical simulations on fracture process in different specimens. From these studies, we can conclude that the present FFTbased analysis is capable to deal with crack initiation and crack growth in composite laminates with high accuracy and efficiency, especially in the cases of matrix/fibre interface debonding and of multi-crack growth.
\end{abstract}

(c) 2011 Elsevier Ltd. All rights reserved.

\section{Introduction}

Currently, the carbon fibre reinforced polymer matrix composite laminates have been increasingly employed in various fields including the airplane, hoses in fluid transport, electricity systems or sportive equipments due to their outstanding performance, combining high stiffness and strength at low density, high-specific energy absorption, excellent fatigue performance and high corrosion resistance. Since the stiffness and strength of an individual layer are much higher in the fibre direction than in the transverse direction, an appropriate design associating the physical and mechanical properties of materials with the geometry shapes is necessary to profit from the fibre performance.

The stiffness degradation is an important response to the damage and crack evolution of fibre-reinforced composite laminates under monotone or cyclic loads. The failure mechanisms of these composites have a complex nature due to their sophisticate microscopic and/or mesoscopic structures. Generally speaking, two prin-

\footnotetext{
* Corresponding author. Address: LSPM, CNRS UPR 3407, Institut Galilée, Université Paris 13, 99 Avenue Jean-Baptiste Clément, 93430 Villetaneuse, France. Tel.: +331494028 89; fax: +33149403938.

E-mail address: jia.li@univ-paris13.fr (J. Li).
}

cipal failure mechanisms can be distinguished: the first one is the bulk damage in the matrix and the fibres and the second is the interface debonding between them. The most widely used theoretical tools in the assessment of these failure mechanisms are the fracture mechanics and damage mechanics.

Over the past decades, the continuum damage mechanics [4] has been widely used to predict the isotropic/anisotropic damage evolution of composites by introducing a phenomenological damage tensor $D$ relevant to the matrix and fibre failure [5-9]. The damage/plasticity coupling non-linear models have also been developed to describe the interactive effect of the plastic deformation on damage properties [10-12]. Combined to different failure criteria [13-17] on the initial failure of composite laminates with fibre principal orientations, the continuum damage mechanics has proven its efficiency on bulk failure assessment. However, the numerical application of this approach on interface debonding between the matrix and fibres is quite difficult due to its small thickness dimension for which very fine element meshing is required.

On the other hand, there is an evolving trend to develop energybased failure criteria in the frame of fracture mechanics. Particularly, the cohesive zone conception $[18,19]$ was adapted to describe the crack propagation in composite materials. The delamination 
mechanisms and crack-bridging mechanisms were thoroughly studied by using the cohesive theory [20-25,3]. These cohesive fracture criteria gain insight into the microscopic damage evolution mechanisms of composites. However, numerical convergence problems may arise from cohesive modelling due to assumed softening properties which require to be solved appropriately as it is implemented into a finite element code. This poses a large challenge to the practical application of the cohesive theory in the damage evolution modelling of composite laminates.

Even though multiple numerical techniques were proposed in the literature, the multiscale progressive failure analysis still meets huge difficulties in incorporating discrete fibre/matrix microscopic modelling and failure mechanisms into damage and failure properties of the whole composite laminates. We believe that alternative methods are useful to enrich the numerical tools in the resolution of such a complicated problem.

In this paper, we propose a non-local fracture model resolved by using the FFT (Fast Fourier Transform) method. A macroscopic failure criterion, in occurrence the maximum stress criterion for simplicity, was used to describe the failure of the matrix and the fibres. This criterion was connected to the Griffith criterion in order to enable it to predict the crack initiation and propagation. A debonding model established on the basis of the Dugdale cohesive concept [3] was adapted for describing the matrix/fibre delamination. The main advantage of the FFT method is its high resolution in discretization of the composite structures such that the matrix/fibre interface can be modelled by a thin layer of interphase. Consequently, some complicated mechanisms such as the matrix/fibre debonding, the propagation of multiple cracks or the crack bridging can directly be simulated.

In this paper, we first recall the FFT-based formulation and the non-local damage model in Sections 2 and 3. In Sections 4 and 5, we describe the adaptation of the Griffith criterion to bulk damage and to interface debonding criteria via the non-local approach. After a short explanation of the numerical algorithm in Section 6, we present in Section 7 several numerical simulations in which the accuracy and the efficiency of the proposed method were examined. Some concluding remarks are given in Section 8.

\section{The FFT-based formulation}

The damage states that develop in brittle or quasi-brittle materials can be evaluated using an extension of an iterative method on the basis of Fast Fourier Transforms (FFT), originally proposed by Moulinec and Suquet [1,2] and Michel et al. [26] for homogenising linear and non-linear composites. The FFT-based formulation for a periodic heterogeneous cell with damage can straightforwardly be written according to the original FFT scheme. By omitting the rigid body motion, the displacement in a periodic cell is split into two parts:

$\mathbf{u}(\mathbf{x})=\mathbf{u} *(\mathbf{x})+\mathbf{E} \cdot \mathbf{x}$

where $\mathbf{x}$ denotes the Cartesian coordinates originated at the geometrical centre of the cell; $\mathbf{u} *(\mathbf{x})$ is the periodically oscillatory part of the displacement with $\int_{\Omega} \mathbf{u} *(\mathbf{x}) d V=\mathbf{0} ; \mathbf{E}$ is the average strain tensor. In the case when the damage field can be expressed by a scalar variable $D(\mathbf{x}) \in[0,1]$, the problem of elasticity for an inhomogeneous elastic composite under periodic boundary conditions writes:

$\boldsymbol{\sigma}(\mathbf{x})=\left[1-D(\mathbf{x})+k_{0}\right] \mathbf{C}(\mathbf{x}):[\boldsymbol{\varepsilon}(\mathbf{u} *(\mathbf{x}))+\mathbf{E}]$

$\operatorname{div} \boldsymbol{\sigma}(\mathbf{x})=0$

$\mathbf{u} *(\mathbf{x})$ is periodic $\forall \mathbf{x} \in \boldsymbol{\Omega}, \boldsymbol{\sigma} . \mathbf{n}$ is anti - periodic $\forall \mathbf{x} \in \partial \boldsymbol{\Omega}$

where $\boldsymbol{\sigma}$ is the Cauchy stress field; $\boldsymbol{\varepsilon}(\mathbf{u} *)$ is the fluctuation term of the strain field in $\Omega$ and satisfies $\int_{\Omega} \boldsymbol{\varepsilon}(\mathbf{u} *(\mathbf{x})) d V=\mathbf{0}$; the damage field $D(\mathbf{x})$ and the stiffness tensor $\mathbf{C}(\mathbf{x})$ are also periodic; $k_{0}$ is a parameter of very small value, it ensures the existence and uniqueness of the numerical solution; $\mathbf{n}$ is the unit outward vector of the cell boundary. This local problem can be resolved by introducing a polarisation stress field $\tau(\mathbf{x})$,

$\boldsymbol{\sigma}(\mathbf{x})=\mathbf{C}^{r}: \boldsymbol{\varepsilon}(\mathbf{u} *)+\tau(\mathbf{x})$

with $\mathbf{C}^{r}$ being the stiffness of a homogeneous reference material and

$\boldsymbol{\tau}(\mathbf{x})=\left(1-D+k_{0}\right) \mathbf{C}:[\boldsymbol{\varepsilon}(\mathbf{u} *)+\mathbf{E}]-\mathbf{C}^{r}: \boldsymbol{\varepsilon}(\mathbf{u} *)$

The solution of (2) can be expressed by means of the periodic Green operator $\boldsymbol{\Gamma}$ associated with $\mathbf{C}^{r}$, namely

$\boldsymbol{\varepsilon}(\mathbf{x})=-\int_{\Omega} \Gamma(\mathbf{x}-\mathbf{y}): \tau(\mathbf{y}) d \mathbf{y}$

By performing the Fourier transformation, this convolution integral is transformed into a direct tensor product:

$\hat{\boldsymbol{\varepsilon}}(\xi)=-\widehat{\Gamma}(\xi): \hat{\tau}(\xi) \quad \forall \xi \neq 0 \quad \hat{\boldsymbol{\varepsilon}}(0)=\mathbf{E}$

where $\hat{\boldsymbol{\varepsilon}}, \hat{\tau}$ and $\widehat{\boldsymbol{\Gamma}}$ are respectively the Fourier transforms of $\boldsymbol{\varepsilon}, \tau$ and $\widehat{\Gamma}, \xi$ denotes the frequencies in Fourier space. When the reference material is isotropic with the Lamé coefficients $\lambda^{r}$ and $\mu^{r}$, the Green operator $\widehat{\Gamma}$ takes the form:

$$
\begin{aligned}
\hat{\Gamma}_{i j k l}= & \frac{1}{4 \mu^{r}|\xi|^{2}}\left(\delta_{k i} \xi_{k} \xi_{l}+\delta_{l i} \xi_{k} \xi_{j}+\delta_{k j} \xi_{l} \xi_{i}+\delta_{l j} \xi_{k} \xi_{i}\right) \\
& -\frac{\left(\lambda^{r}+\mu^{r}\right)}{\mu^{r}\left(\lambda^{r}+2 \mu^{r}\right)} \frac{\xi_{i} \xi_{j} \xi_{k} \xi_{l}}{|\xi|^{4}}
\end{aligned}
$$

The anti-transforming of (6) gives the strain field of the problem. However, since the polarisation stress field is a priori unknown for a damaged heterogeneous material, iterative procedure has to be used to obtain a compatible strain field and then a stress field in equilibrium.

In the present work, only the plane problems are considered. For plane strain, all the precedent formulas are directly valid for bi-dimensional simulations. For plane stress, the same equations in plane strain can be kept if the Lamé coefficient $\lambda$ is replaced by $\lambda \frac{2 \mu}{\lambda+2 \mu}$.

\section{Non-local damage model}

Numerous continuum damage models exist in the literature to describe the progressive degradation of materials. The accuracy of the classical damage models often depend on the finite element discretization in their numerical implementation. In order to overcome this shortcoming, various regularization methods have been proposed. Among these methods, the so-called non-local approaches are widely used. The basic idea of this approach consists in replacing the local damage driving force, an effective stress $\sigma_{e}$ for example, by its weighted average over a representative volume $V$ [27]:

$\tilde{\sigma}_{e}(\mathbf{x})=\frac{1}{\int_{V} \alpha(\mathbf{x}-\mathbf{y}) d \mathbf{y}} \int_{V} \alpha(\mathbf{x}-\mathbf{y}) \sigma_{e}(\mathbf{y}) d \mathbf{y}$

In the literature, $\alpha$ is often taken as the Gaussian function. In the present work, a cone-shape weighting function is used in the place of the Gaussian function for its periodic feature required in FFT calculations. This function writes:

$\alpha(r)=\left\{\begin{array}{cc}0 & r>R \\ 1-\frac{r}{R} & r \leqslant R\end{array}\right.$

where $r=\|\mathbf{x}-\mathbf{y}\| ; R$ is the radius of non-local action, representing a material characteristic length which defines the size of interaction zone for failure processes. 


\section{Matrix and fibre fracture}

In this study, the matrix and fibre layers are considered as isotropic linear elastic materials. The extension of the present model to anisotropic materials does not present particular difficulties and will be developed later. In this section, the non-local damage model proposed by Li et al. [28] is briefly presented, as follows.

We assume, for simplicity, that the material failure obeys the maximum principal stress criterion under regular stress field. This criterion cannot be directly applied to cracked materials due to the stress singularity near the crack tips. However, it can be related to a crack growth criterion through a non-local approach. Thus, we write the non-local maximum principal stress criterion as follows:

$D= \begin{cases}0 & \tilde{\sigma}_{1}<\sigma_{c} \\ 1 & \tilde{\sigma}_{1} \geqslant \sigma_{c}\end{cases}$

where $\sigma_{c}$ is the ultimate stress of the material, $\tilde{\sigma}_{1}$ is the non-local first principal stress. We assume that the near-tip stress field is governed by the Williams asymptotic expansion [29], therefore. For a mode-I loaded crack, the non-local near-tip first principal stress writes:

$$
\begin{aligned}
\tilde{\sigma}_{1}(r, \theta) & =\frac{1}{\int_{0}^{R} \int_{-\pi}^{\pi}\left(1-\frac{r^{\prime}}{R}\right) r^{\prime} d r^{\prime} d \theta^{\prime}} \int_{0}^{R} \int_{-\pi}^{\pi}\left(1-\frac{r^{\prime}}{R}\right) \sigma_{1}(r, \theta) r^{\prime} d r^{\prime} d \theta^{\prime} \\
& =\frac{3}{\pi R^{2}} \int_{0}^{R} \int_{-\pi}^{\pi}\left(1-\frac{r^{\prime}}{R}\right) \frac{K_{I}}{\sqrt{2 \pi r}}\left(1+\cos \frac{\theta}{2}\right)\left|\sin \frac{\theta}{2}\right| r^{\prime} d r^{\prime} d \theta^{\prime}
\end{aligned}
$$

where $K_{I}$ is the stress intensity factor. Under mode I loading, the maximal non-local principal stress is located at a point on the crack axis near the crack tip $r=r_{0}, \theta=0$ due to the symmetry. We assume that $r_{0}$ is small such that the stress at its vicinity is still governed by the crack-tip asymptotic field.

On the one hand, according to the damage criterion (10), the element at $\left(r=r_{0}, \theta=0\right)$ is broken when $\tilde{\sigma}_{1} \geqslant \sigma_{c}$. On the other hand, from the Griffith-Irwin criterion of fracture [30,31], the crack grows when $K_{I} \geqslant K_{I c}$, where $K_{I c}$ is the critical stress intensity factor. This class of strength-toughness crack growth criteria was successively used in the prediction of crack initiation near a V-notch tip or a hole $[32,33]$. This condition permits us to determine the non-local action radius $R$ and the location of the most loaded point $\left(r=r_{0}, \theta=0\right)$ by resolving numerically the following equation:

$$
\begin{aligned}
f(R)= & \sigma_{c}-\max _{r_{0}} \int_{0}^{R} \int_{-\pi}^{\pi} \frac{3}{\pi R^{2}}\left(1-\frac{r^{\prime}}{R}\right) \\
& \times \frac{K_{I c}}{\sqrt{2 \pi r}}\left(1+\cos \frac{\theta}{2}\right)\left|\sin \frac{\theta}{2}\right| r^{\prime} d r^{\prime} d \theta^{\prime}=0
\end{aligned}
$$

with $r=\sqrt{\left(r_{0}+r^{\prime} \cos \theta^{\prime}\right)^{2}+\left(r^{\prime} \sin \theta^{\prime}\right)^{2}} \quad \tan \theta=\frac{r^{\prime} \sin \theta^{\prime}}{r_{0}+r^{\prime} \cos \theta^{\prime}}$

By taking $K_{I c} / \sigma_{c}=0.5 \sqrt{m m}$, for example, we can find $R=0.105 \mathrm{~mm}, r_{0}=0.03 \mathrm{~mm}$. For mode II and mixed modes, the non-local action radius $R$ is slightly larger. In general, the mode I crack propagation is the most frequent fracture mode in brittle materials. Therefore, for simplicity, the value of $R$ obtained from a mode I crack is used for all the mixed-mode cracks in the present work, this leads to slightly conservative critical loads at fracture comparing with the criterion $G \geqslant G_{c}=K_{I c}^{2} / E$, where $G$ and $G_{c}$ are respectively the energy release rate of the crack and its critical value; $E$ is the Young modulus of the material. As a result, the non-local damage criterion (10) is equivalent to the Griffith-Irwin criterion for crack propagation and to the maximal principal stress criterion for failures in non-cracked structures. Consequently, it can be used to predict the crack initiation as well as the crack propagation. In practice, we just need to find the point where the non- local principal stress is maximal: this point is broken when the non-local stress attaints the material strength.

\section{Interface debonding}

In the present study, the interface debonding is modelled by fracture of a thin layer of interphase zone. This approach is numerically costly but can provide more detailed information on matrix/ fibre debonding compared with the pure theoretical interface assessments.

Consider now a bi-material interface lying along the $x$-axis, the material 1 occupies the half space $y>0$ while the material 2 occupies the half space $y<0$. The interface is modelled in the present study by a thin layer of interphase whose fracture can be described by a damage criterion similar to (10):

$D= \begin{cases}0 & \tilde{\sigma}_{e} / \sigma_{c}<1 \\ 1 & \tilde{\sigma}_{e} / \sigma_{c} \geqslant 1\end{cases}$

Here we have replaced the non-local maximum principal stress $\tilde{\sigma}_{1}$ by a non-local effective stress $\tilde{\sigma}_{e}$. In fact, due to the thin geometry of the interphase zone, we have to distinguish the fracture due the interface debonding from that due to $\sigma_{x x}$. Thus, the nominal effective stress should be understood as the maximum of these two failure origins:

$\tilde{\sigma}_{e} / \sigma_{c}=\max \left(\tilde{\sigma}_{e}^{\text {debond }}\left(\tilde{\sigma}_{y y}, \tilde{\sigma}_{x y}\right) / \sigma_{c}, \tilde{\sigma}_{x x} / \sigma_{c}\right)$

In interface fracture mechanics, the interface crack growth is usually predicted by comparing the energy release rate to its critical value. In general, the interface toughness is highly influenced by the mode mixity near the crack tip with respect to the interface plan [34,35]. Some empirical interface debonding criteria accounting for this influence have been proposed in the literature $[21,36]$. Based on the Dugdale cohesive model, Li [3] has obtained the critical energy release rate $G_{c}$ for interfacial crack growth that can be written, for a sufficiently long interface crack, as follows:

$G_{c}=G_{I c} \frac{\left[1+\left(\tau_{c} / \sigma_{c}\right)^{2}\right]\left(1+\tan ^{2} \Psi\right)}{1+\left(\tau_{c} / \sigma_{c}\right)^{2}+\left[1+\left(\sigma_{c} / \tau_{c}\right)^{2}\right] \tan ^{2} \Psi}$

where $G_{I c}$ is the critical energy release rate for pure mode I debonding; $\sigma_{c}$ and $\tau_{c}$ are respectively the critical interface cohesive forces under normal tension and pure shear; $\tan \Psi=q / p$ where $p$ and $q$ are respectively the normal traction and the shear force acting on the crack lips in the cohesive zone. Consequently, $\Psi$ represents the mixity angle at the crack tip. For an interface crack between two dissimilar materials, the traction along the interface ahead of the crack tip writes:

$\left(\sigma_{y y}+i \sigma_{x y}\right)_{\text {interface }}=\frac{K r^{i \varepsilon}}{\sqrt{2 \pi r}}$

where $r$ is the distance between the crack tip and the considered point; $K=\left(K_{I}+i K_{I I}\right) \cosh (\pi \varepsilon)$ is a complex stress intensity factor which uniquely characterise the singular field [37]; $\varepsilon$ is the oscillatory index. The energy release rate for an interfacial crack tip is [38]:

$G=\left(\frac{\kappa_{1}+1}{\mu_{1}}+\frac{\kappa_{2}+1}{\mu_{2}}\right) \frac{K \bar{K}}{16 \cosh ^{2}(\pi \varepsilon)}$

with

$\kappa_{1}=\left(3-v_{1}\right) /\left(1+v_{1}\right) \quad \kappa_{2}=\left(3-v_{2}\right) /\left(1+v_{2}\right)$ for plane stress

$\kappa_{1}=3-4 v_{1} \quad \kappa_{2}=3-4 v_{2}$ for plane strain

$\mu_{1}=\frac{E_{1}}{2\left(1+v_{1}\right)} \quad \mu_{2}=\frac{E_{2}}{2\left(1+v_{2}\right)}$ 
where $E_{1}, v_{1}, E_{2}$ and $v_{2}$ are respectively the Young moduli and the Poisson ratios of the two materials. We can calculate the non-local interfacial traction at a point $r_{0}$ ahead of the crack tip by

$\left(\tilde{\sigma}_{y y}+i \tilde{\sigma}_{x y}\right)\left(r=r_{0}\right)=\frac{1}{\int_{-r_{0}}^{R}\left(1-\left|r^{\prime} / R\right|\right) d r^{\prime}} \int_{-r_{0}}^{R} \frac{K\left(r_{0}+r^{\prime}\right)^{i \varepsilon}}{\sqrt{2 \pi\left(r_{0}+r^{\prime}\right)}}\left(1-\left|\frac{r^{\prime}}{R}\right|\right) d r^{\prime}$

The maximal values of these non-local stresses are found at $r_{0}=0$. Thus

$\left(\tilde{\sigma}_{y y}+i \tilde{\sigma}_{x y}\right)(r=0)=\frac{C}{\sqrt{2 \pi R}}\left(K_{I}^{\prime}+i K_{I I}^{\prime}\right) \cosh (\pi \varepsilon)$

with

$\begin{array}{ll}K_{I}^{\prime}=K_{I} \cos (\alpha-\varepsilon \ln R)+K_{I I} \sin (\alpha-\varepsilon \ln R) & K_{I I}^{\prime}=K_{I I} \cos (\alpha-\varepsilon \ln R)-K_{I} \sin (\alpha-\varepsilon \ln R) \\ \tan \alpha=\frac{2 \varepsilon}{3 / 4-\varepsilon^{2}} & C=\frac{2}{\sqrt{\left(3 / 4-\varepsilon^{2}\right)^{2}+\varepsilon^{2}}}\end{array}$

Without introducing confusions, hereafter $\left(\tilde{\sigma}_{y y}+i \tilde{\sigma}_{x y}\right)(r=0)$ will be replaced by $\tilde{\sigma}_{y y}+i \tilde{\sigma}_{x y}$ for conciseness. The non-local action radius $R$ is calculated by considering the pure normal tension mode according to the arguments mentioned in Section 4. In this case, the interfacial crack grows when the non-local strength and toughness criteria $\tilde{\sigma}_{y y} \geqslant \sigma_{c}$ and $K_{I}^{\prime} \geqslant K_{I c}$ are both satisfied, thus we obtain:

$R=\frac{C^{2} \cosh ^{2}(\pi \varepsilon)}{2 \pi}\left(\frac{K_{I c}}{\sigma_{c}}\right)^{2}$

This relation also permits us to connect the non-local stresses to the energy release rate. According to (17) and (19), we have

$G=\left(\frac{\kappa_{1}+1}{\mu_{1}}+\frac{\kappa_{2}+1}{\mu_{2}}\right) \frac{2 \pi R}{C^{2}} \frac{\left(\tilde{\sigma}_{y y}^{2}+\tilde{\sigma}_{x y}^{2}\right)}{16 \cosh ^{2}(\pi \varepsilon)}$

From (17), the critical energy release rate for interfacial crack growth under pure mode-I loading is defined as follows:

$G_{I c}=\left(\frac{\kappa_{1}+1}{\mu_{1}}+\frac{\kappa_{2}+1}{\mu_{2}}\right) \frac{K_{I c}^{2}}{16}$

Thus according to (15) and (23), the interface crack growth criterion $G \geqslant G_{c}$ under mixed mode loading is equivalent to:

$\tilde{\sigma}_{e}^{\text {debond }} \geqslant \sigma_{c} \sqrt{\frac{\left[1+\left(\tau_{c} / \sigma_{c}\right)^{2}\right]\left(1+\tan ^{2} \Psi\right)}{1+\left(\tau_{c} / \sigma_{c}\right)^{2}+\left[1+\left(\sigma_{c} / \tau_{c}\right)^{2}\right] \tan ^{2} \Psi}}$

where

$\tilde{\sigma}_{e}^{\text {debond }}=\sqrt{\tilde{\sigma}_{y y}^{2}+\tilde{\sigma}_{x y}^{2}}, \quad \tilde{\sigma}_{y y}=0$ if $\quad \tilde{\sigma}_{y y}<0$

is the effective non-local traction at the crack tip. The non-local mode mixity $\tan \Psi=q / p$ is reasonably replaced by $\tan \Psi=$ $\tilde{\sigma}_{x y} / \tilde{\sigma}_{y y}$. Thus the nominal effective non-local stress in the damage criterion (13) writes

$\tilde{\sigma}_{e}=\max \left(\frac{\tilde{\sigma}_{e}^{\text {debond }}}{\alpha_{1}}, \frac{\tilde{\sigma}_{x x}}{\alpha_{2}}\right)$

where $\alpha_{1}$ and $\alpha_{2}$ are strength factors defined as follows

$$
\begin{gathered}
\alpha_{1}=\sqrt{\frac{\left[1+\left(\tau_{c} / \sigma_{c}\right)^{2}\right]\left(1+\tan ^{2} \Psi\right)}{1+\left(\tau_{c} / \sigma_{c}\right)^{2}+\left[1+\left(\sigma_{c} / \tau_{c}\right)^{2}\right] \tan ^{2} \Psi}} \\
\alpha_{2}>1
\end{gathered}
$$

It is clear that the non-local effective stress $\tilde{\sigma}_{e}^{\text {debond }}$ is the main responsible of the interface debonding. The criterion $\tilde{\sigma}_{x x} / \alpha_{2} \sigma_{c} \geqslant 1$ is less important but numerically necessary to ensure the rupture of the interphase under pure tension $\sigma_{x x}$. The parameter $\alpha_{2}$ should be sufficiently large such that the non-local effective stress $\tilde{\sigma}_{e}^{\text {debond }}$ remains predominant in interface failure.

As the interface is considered as an interphase zone, geometrical and material constants have to be attributed to it. In the case of the joins formed by glue, welding or cement between two elements, an interphase zone does exist and we can just use the geometrical and mechanical parameters of the considered join in the modelling. In the case of the direct adhesion between the matrix and the fibres, the effective Young's modulus $E_{i}$ and Poisson's ratio $v_{i}$ of the interphase can be determined by considering the energy release rate. For a crack in the interphase, the energy release rate at a crack tip writes

$$
G=\frac{K_{I}^{2}+K_{I I}^{2}}{E^{\prime}}\left\{\begin{array}{cl}
E^{\prime}=E_{i} & \text { for plane stress } \\
E^{\prime}=\frac{E_{i}}{1-v_{i}^{2}} & \text { for plane stress }
\end{array}\right.
$$

Comparing (28) to (17), the effective Young's modulus of the interphase is given by:

$E_{i}=\frac{2 E_{1} E_{2}}{E_{1}+E_{2}}$

and the Poisson's ratio by

$$
v_{i}=\sqrt{\frac{E_{2} v_{1}^{2}+E_{1} v_{2}^{2}}{E_{1}+E_{2}}}
$$

Interface strength and toughness parameters $G_{I c}, \sigma_{c}$ and $\tau_{c}$ can be obtained by appropriate experiments.

The thickness of the interphase is an important parameter in the fracture assessment. It should be small enough within the limit of the numerical discretization in order to ensure a sufficient accuracy. We did not attempt to give a quantitative estimation for the thickness in the present work. Detailed analysis on its role in interface fracture can be investigated in future works.

\section{Numerical algorithm}

The proposed FFT fracture model was coded by using the programming language MATLAB. First, this language benefits from its complete package for FFT analyses; second, the numerical non-local integration (8) can directly be performed by using the convolution functions provided in the software. Since a composite consists of several phases, the non-local volume used in the calculation of the non-local stresses at a point (Eq. (8)) should only contain the same phase as the considered point.

In the present work, the initial cracks and their growth are represented by domains with very low material stiffness. For example, we can set $E_{\text {damaged }}=k_{0} E$ where $E_{\text {damaged }}$ and $E$ are respectively Young's moduli of cracked zone and non-damaged material. The parameter $k_{0}$ is numerically very small.

In the case of very high contrast between materials, the initial FFT method $[1,2]$ has difficulty to converge. This difficulty can generally be overcome by making use of an augmented Lagrangian method [26]. Nevertheless, $k_{0}=0$ represents an infinite material contrast between damaged and non-damaged materials and as a consequence, leads to a too low convergence speed. We found that $k_{0}=10^{-5}$ represents a good compromise between the computational efficiency and the numerical accuracy.

Since a material element is linearly elastic before its complete failure, therefore, the crack propagation evaluation is very similar to that adopted in the linear elastic fracture mechanics: An elastic calculation is first carried out for the cracked structure, and then small crack propagation and the corresponding remote load are determined according to a suitable criterion. This procedure is then 


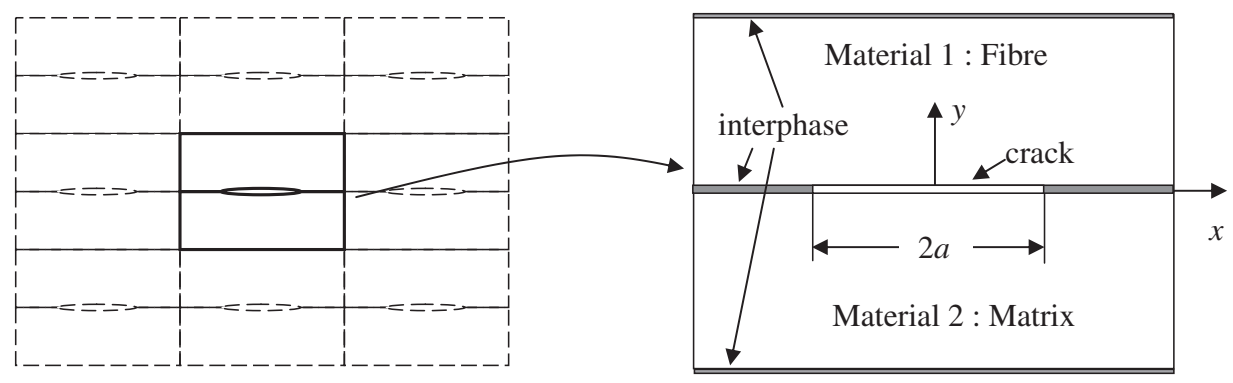

Fig. 1. Basic periodic bi-material cells containing an interfacial crack.

repeated after each small crack progression in the structure. Accordingly, the following algorithm is coded:

1. Resolve the elasticity problem (2) by using the FFT method.

2. Calculate the non-local effective stresses and find the position $\mathbf{x}_{\text {damaged }}$ where the non-local effective stress is maximal.

3. Calculate the remote load for crack growth according to (10) if $\mathbf{x}_{\text {damaged }}$ is located in the matrix or the fibres, (13) if $\mathbf{x}_{\text {damaged }}$ is located in the interphases.

4. Set $D=1-k_{0}$ for pixels in a small zone around $\mathbf{x}_{\text {damaged }}$, the radius of this spot $r_{d}$ should be small enough in order to ensure that the broken zones form a continuously growing crack. When this spot covers several phases, we take precautions such that the broken zone contains only points of the same phase as that of the point $\mathbf{x}_{\text {damaged }}$.

5. Repeat this algorithm until a desired crack growth is achieved.

\section{Numerical results and discussions}

In this section, we present the results several numerical simulations in order to verify the validity and the performance of the present FFT fracture model in predicting the crack evolution in composite laminates with interface debonding.

\subsection{Crack growth in matrix and fibres}

In Li et al. [28], the prediction of the crack growth in a 2D bulk body by means of the FFT damage model described in Section 4 has been compared with that by using the conventional linear fracture mechanics. The principal conclusions of these studies are:

1. The proposed non-local damage model is equivalent to the criterion $K_{I} \geqslant K_{I c}$ for mode I cracks.

2. The crack growth prediction is independent of the FFT grid resolution if the discretization is fine enough.

3. The proposed fracture model is capable to predict crack initiation as well as crack growth.

The numerical results show that the FFT damage model is highly accurate and efficient, particularly in multi-cracking simulations.

\subsection{Interface crack growth}

Now let us consider a 2D plane strain periodic cell formed by two dissimilar materials containing a central interface crack. The dimension of the basic cell is $10 \times 5 \mathrm{~mm}^{2}$ with a central interface crack $2 a$ of different sizes, namely $a=0,0.1,0.2,0.3,0.5,0.75,1$, $1.25,1.5$ and $2 \mathrm{~mm}$, here $a=0$ represents a plate without crack. The interface was simulated by an interphase of $0.025 \mathrm{~mm}$. The cell was discretized using a grid of regularly spaced Fourier points of
Table 1

Material constants of the interphase.

\begin{tabular}{cllll}
\hline$E_{\text {fibre }} / E_{\text {matrix }}$ & $E_{i} / E_{\text {matrix }}$ & $v_{\text {matrix }}=v_{\text {fibre }}=v_{i}$ & $\varepsilon$ & $R_{i}$ \\
\hline 1 & 1 & 0.22 & 0 & 0.2829 \\
2 & 1.333 & 0.22 & 0.0383 & 0.2852 \\
5 & 1.667 & 0.22 & 0.0777 & 0.2923 \\
10 & 1.818 & 0.22 & 0.0963 & 0.2973 \\
\hline
\end{tabular}

$800 \times 400$ pixels. Thus the interphase contains 2 FFT pixels in its thickness. The geometry of the basic cell is drawn in Fig. 1.

Let the material 1 in $y>0$ be the fibre and harder and the material 2 in $y<0$ be the matrix and softer. Several material combinations were examined. The Young modulus $E_{i}$ and the Poisson ratio $v_{i}$ of the interphase were calculated from (29) and (30). By assuming that the toughness/strength ratio of the interphase $K_{\text {Ic }} / \sigma_{c}=0.5 \sqrt{\mathrm{mm}}$, the non-local action radius $R_{i}$ for the interphase can be calculated by (21). The material parameters of such a bimaterial are listed in Table 1 for different bi-materials.

The critical energy release rate for a pure mode I crack in interphase is given by, according to (28):

$G_{I c}=\frac{K_{l c}^{2}}{E^{\prime}}\left\{\begin{array}{cl}E^{\prime}=E_{i} & \text { for plane stress } \\ E^{\prime}=\frac{E_{i}}{1-v_{i}^{2}} & \text { for plane stress }\end{array}\right.$

With these parameters and by setting the interphase ultimate stress ratio $\tau_{c} / \sigma_{c}=10$, the interphase toughness under mixed mode loading can directly be obtained by using the criterion (13).

This simple bi-material cell containing interfacial cracks was used to evaluate the accuracy of the present FFT model, namely:

\subsubsection{Comparison with the Griffith criterion $G \geqslant G_{c}$}

For the considered interfacial cracks, the stress intensity factors can be approximated by those for an interfacial crack between two semi-infinite panels of dissimilar materials when the crack length is small compared with the size of the basic cell. For the interface crack of length $2 a$ subjected to remotely uniform stress $\sigma_{y y}^{\infty}$ and $\sigma_{x y}^{\infty}$, the stress intensity factors at the right-hand crack tip are given by [39]:

$K=K_{I}+i K_{I I}=\left(\sigma_{y y}^{\infty}+i \sigma_{x y}^{\infty}\right)(1+2 i \varepsilon)(2 a)^{-i \varepsilon} \sqrt{\pi a}$

The energy release rate can be calculated from (17), namely:

$G=\left(\frac{\kappa_{1}+1}{\mu_{1}}+\frac{\kappa_{2}+1}{\mu_{2}}\right) \frac{\left(\left(\sigma_{y y}^{\infty}\right)^{2}+\left(\sigma_{x y}^{\infty}\right)^{2}\right)\left(1+4 \varepsilon^{2}\right) \pi a}{16 \cosh ^{2}(\pi \varepsilon)}$

Therefore, the theoretical toughness of the interface crack can easily be evaluated according to the Griffith criterion $G \geqslant G_{c}$. In the following, we will compare the theoretical toughness with the numerical FFT results of the present non-local model. 


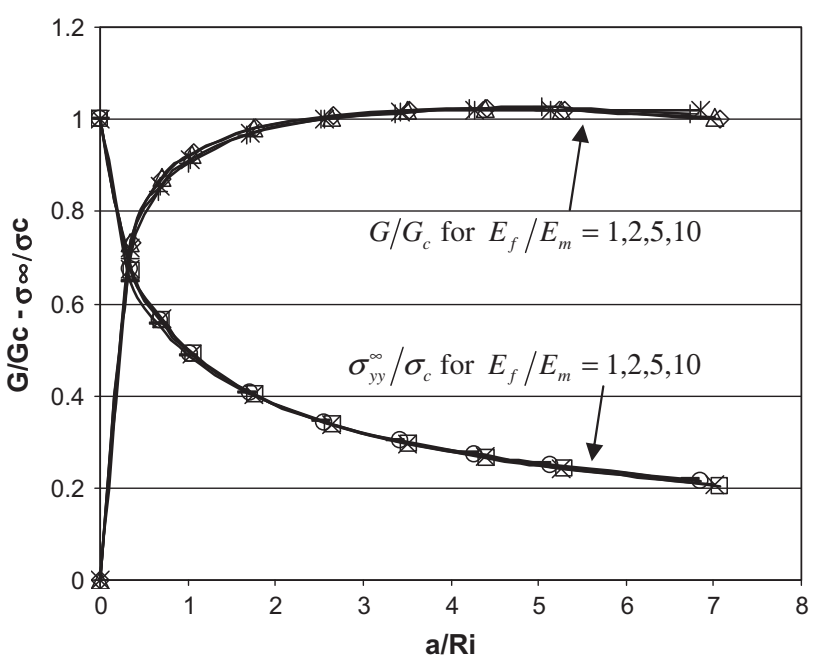

Fig. 2. Normalised energy release rate $G / G_{c}$ and normalised remote stresses $\sigma_{y y}^{\infty} / \sigma_{c}$ as function of normalised semi-crack length $a / R_{i}$ for interface cracks at fracture.

In FFT simulations, the remote load was applied by imposing an average strain $E_{11}=E_{12}=0$ and $E_{22}>0$. The remote stress at fracture was calculated by averaging the tensile stress over the basic cell, $\sigma_{22}^{\infty}=\Sigma_{22}=\frac{1}{V} \int_{\Omega} \sigma_{22} d V$. By using the damage criterion (13), the critical remote stress corresponding to the crack growth was calculated by using the present non-local model. According to (30), this remote load allows the calculation of the energy release rate $G$ at fracture. At the same time, the crack-tip mode mixity can be evaluated by the non-local stresses at the broken point according to $\tan \Psi=\tilde{\sigma}_{12} / \tilde{\sigma}_{22}$, thus the critical energy release rate $G_{c}$ is obtained from (15) and (31). Theoretically,

$G / G_{c}$ should be unity at crack growth. In Fig. 2, we plot $G / G_{c}$ and $\sigma_{22}^{\infty} / \sigma_{c}$ as function the normalised crack length $a / R_{i}$ for different material combinations. From Fig. 2, we can remark the following points:

- The present non-local interphase damage model is equivalent to the criterion $G / G_{C}=1$ with quite a good accuracy.

- When the interface crack length tends to zero, the proposed crack growth criterion is degenerated to the maximum stress criterion.

\subsubsection{Comparison with experimental data of Liechti and Chai [34]}

Amongst many test data on interface crack toughness, the experimental work carried out by Liechti and Chai [34] may be one of the most accurate studies due to the optical interferometry technique used in their crack opening measurements. The specimen that they used was an edge-cracked epoxy/glass biomaterial strip. The specimen is quite thick such that it can be considered as a plane strain structure. The material properties of the specimens are:

- Glass: $E_{1}=69,000 \mathrm{MPa}, v_{1}=0.2$.

- Epoxy: $E_{2}=2030 \mathrm{MPa}, v_{2}=0.36$.

- Oscillatory index: $\varepsilon=0.061$.

- Interface: Critical energy release rate for mode I fracture is $G_{I c}=3.3 \mathrm{~J} / \mathrm{m}^{2}$.

It is clear that the real experimental specimens cannot directly be simulated by the FFT method as this method deals with exclusively periodic structures. However, due to the autonomy of the crack-tip fields, we can still use the 2D plane strain periodic cell shown in Fig. 1 to assess the crack growth of the real specimens

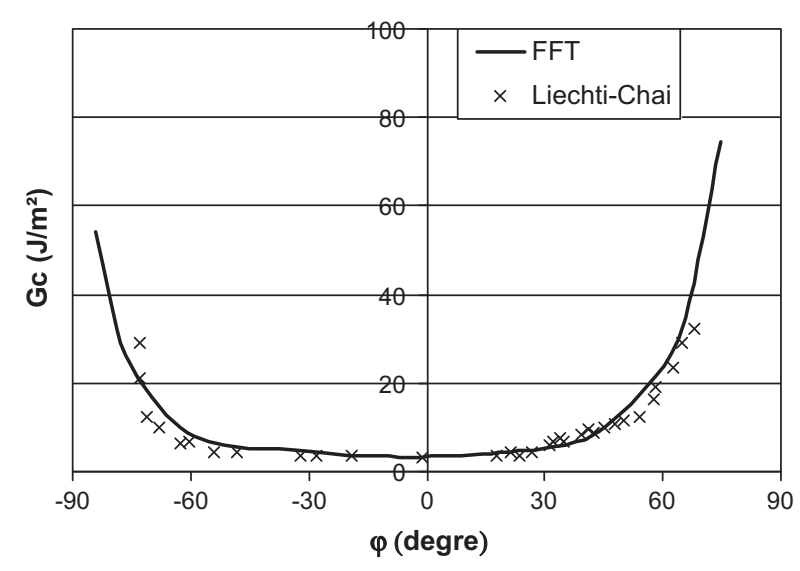

Fig. 3. Comparison between the numerical prediction and experimental data in [34].

if the energy release rates are identical in the two structures. In the present numerical study, the interface is simulated by an interphase with a thickness of $0.025 \mathrm{~mm}$. Some material constants of the interphase can be calculated according to Eqs. (29) and (30), namely,

$E_{i}=3944 \mathrm{MPa}, v_{i}=0.3564$; The critical stress intensity factor is therefore $K_{I c}=\sqrt{G_{I c} E_{i} /\left(1-v_{i}^{2}\right)}=3.86 \mathrm{MPa} \sqrt{m m}$. Apart from these parameters, we assume that the toughness/strength ratio of the interphase $K_{I c} / \sigma_{c}=0.5 \sqrt{\mathrm{mm}}$, the ultimate stress ratio $\tau_{c} / \sigma_{c}=10$. Thus the non-local action radius of the interphase is $R_{i}=0.282 \mathrm{~mm}$ according to (21).

The critical energy release rates were calculated for this basic cell under different mixed mode loads according to the non-local criterion (13). In Fig. 3, we compare the numerical predictions thus obtained with the experimental results in [34]. The crack-tip mode mixity in this figure is calculated on the basis of a reference length of $100 \mu \mathrm{m}$ as in [34], i.e., $\varphi=\arctan \frac{K_{I I}}{K_{I}}+\varepsilon \ln (0.1)$ according to the definition in (16), with $K_{I}$ and $K_{I I}$ calculated from (32).

From Fig. 3, we can notice that the numerical predictions for interface crack growth agree very well with the experimental data. The U-shaped asymmetric mixed mode interface toughness was correctly reproduced.

\subsection{Fracture in composite laminates}

Through the examples above-presented, we can confirm that the proposed non-local crack growth models are highly accurate and efficient for the prediction of crack propagation in bulk materials and along bi-material interfaces. Another notable advantage of the present method is its capacity to evaluate multiple crack growth as it does not require the calculation of the energy release rate at each crack tip. Since the proposed non-local damage model is equivalent to the Griffith crack growth criterion, the failure of the most loaded point in the body corresponds to the growth of the most loaded crack. In the following examples, we will illustrate this capacity of the proposed models by simulating the fracture evolution in composite laminates, which were frequently studied in the literature.

Let us consider a plane strain composite laminate cell of dimension $20 \times 18.2 \mathrm{~mm}^{2}$ formed by three phases: matrix, fibres and interfaces. The thicknesses of these layers are: $0.625 \mathrm{~mm}$ for matrix, $0.625 \mathrm{~mm}$ for fibres and $0.025 \mathrm{~mm}$ for interphases. The entire cell was divided into pixels by a $400 \times 728$ Fourier grid such that an interphase layer is represented by a row of Fourier points, a matrix layer and a fibre layer are represented each by 25 rows of Fou- 
Table 2

Material constants of the components.

\begin{tabular}{|c|c|c|c|c|c|}
\hline & $E(\mathrm{MPa})$ & $v$ & $\sigma_{c}(\mathrm{MPa})$ & $K_{I c}(\mathrm{MPa} \sqrt{\mathrm{mm}})$ & $R(\mathrm{~mm})$ \\
\hline Matrix (epoxy) & 5100 & 0.35 & 100 & 50 or 200 & 0.105 or 1.486 \\
\hline Fibre (carbon) & 210,000 & 0.27 & 1400 & 700 & 0.105 \\
\hline Interphase & 10,451 & 0.3483 & 40 or 60 & 20 or 30 & 0.2906 \\
\hline
\end{tabular}

rier points. A small initial crack of length $2 a=0.625 \mathrm{~mm}$ normal to the fibre direction is located in a matrix layer at the cell centre. Each layer of the composite is assumed to be linearly elastic and isotropic. The material parameters of each component are listed in Table 2.
The external loads can be applied by imposing average stresses $\Sigma_{11}>0, \Sigma_{22}=\Sigma_{12}=0$ or average strains $E_{11}>0, E_{22}=E_{12}=0$. The FFT simulations were carried out step by step with small crack growth (about $0.1 \mathrm{~mm}$ ) at each step until the full failure of the cell.

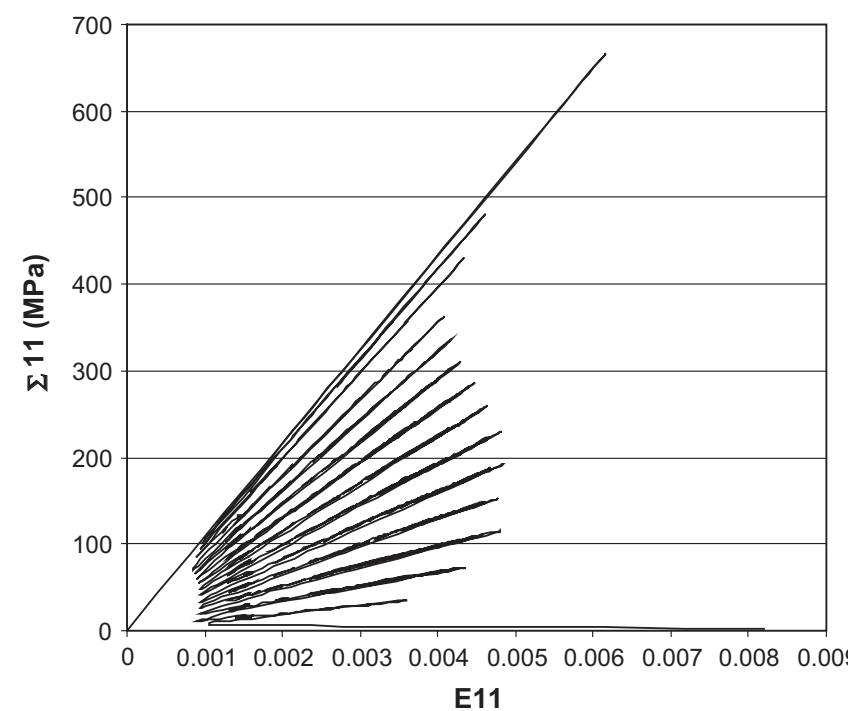

(a)

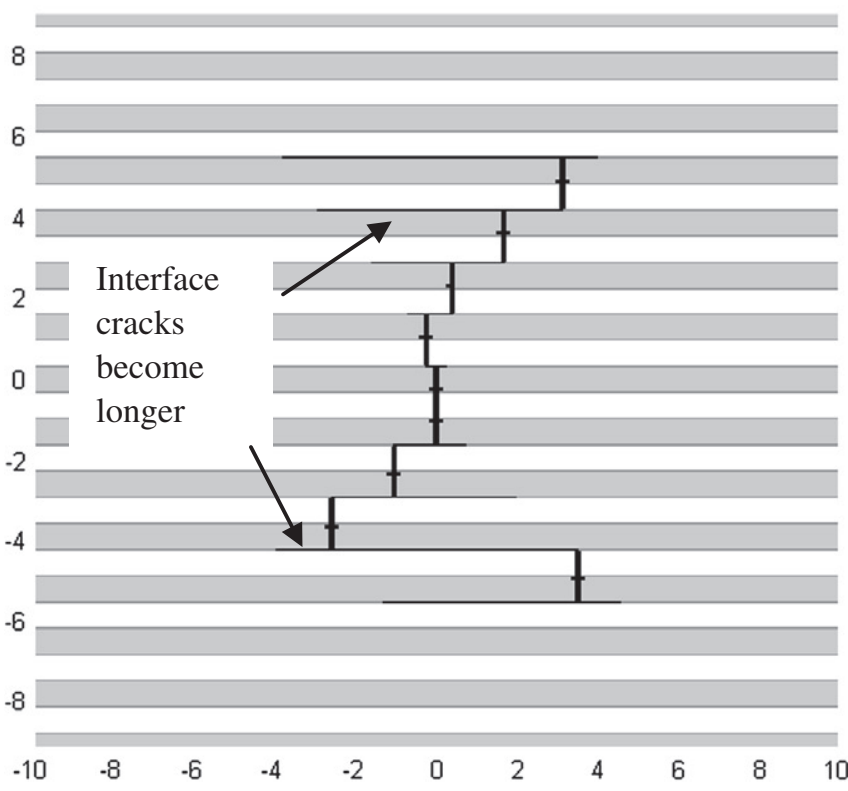

(c)

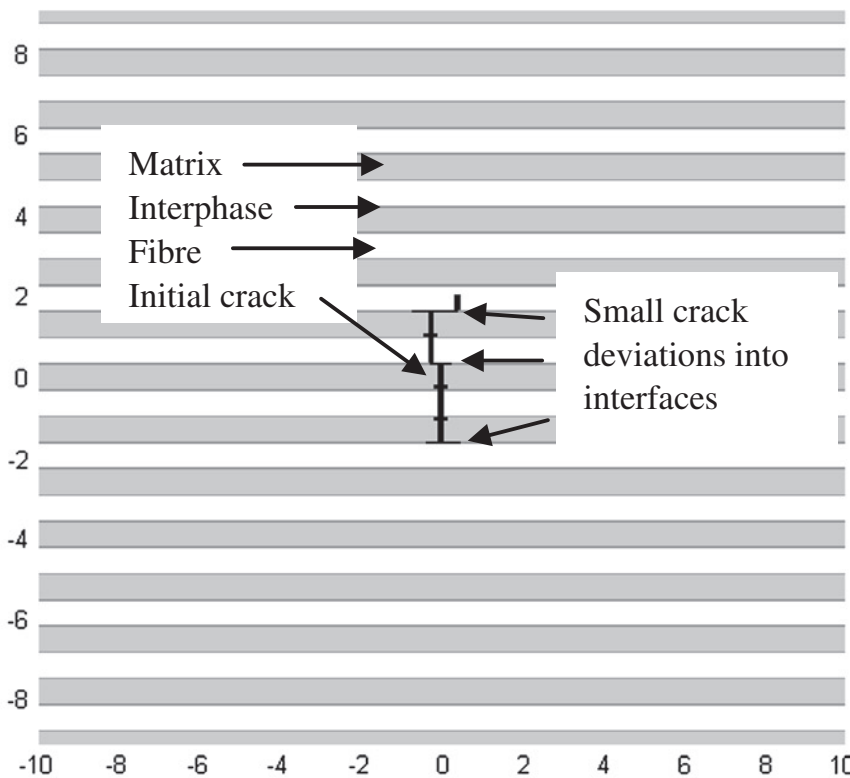

(b)

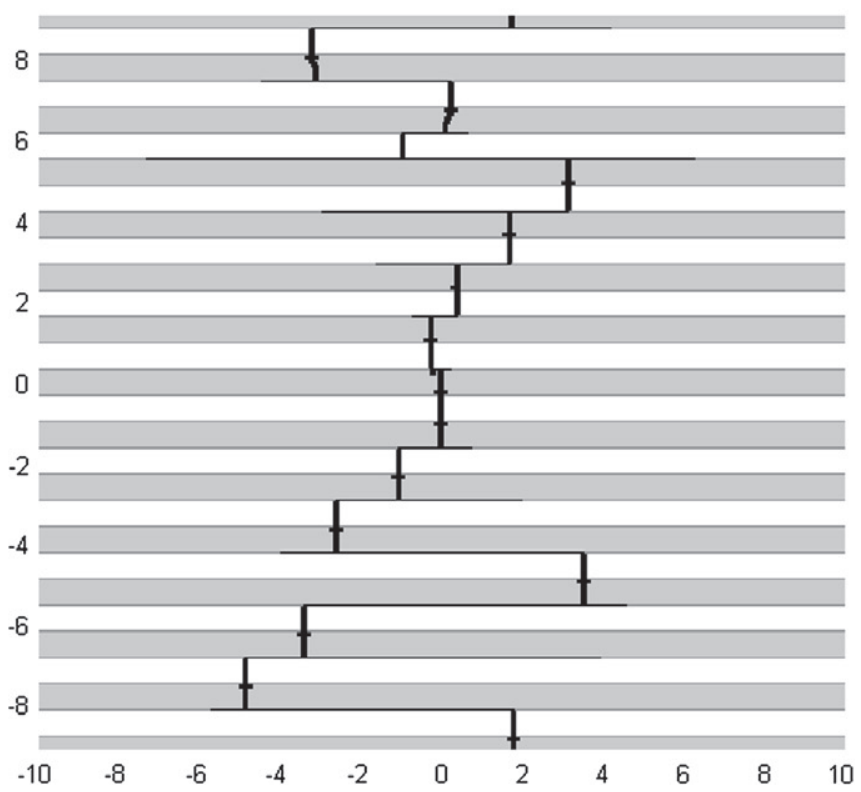

(d)

Fig. 4. Global response (a) and fracture patterns at the beginning (b), the middle (c) and the end (d) of the failure process of the basic cell under uniaxial tension. 


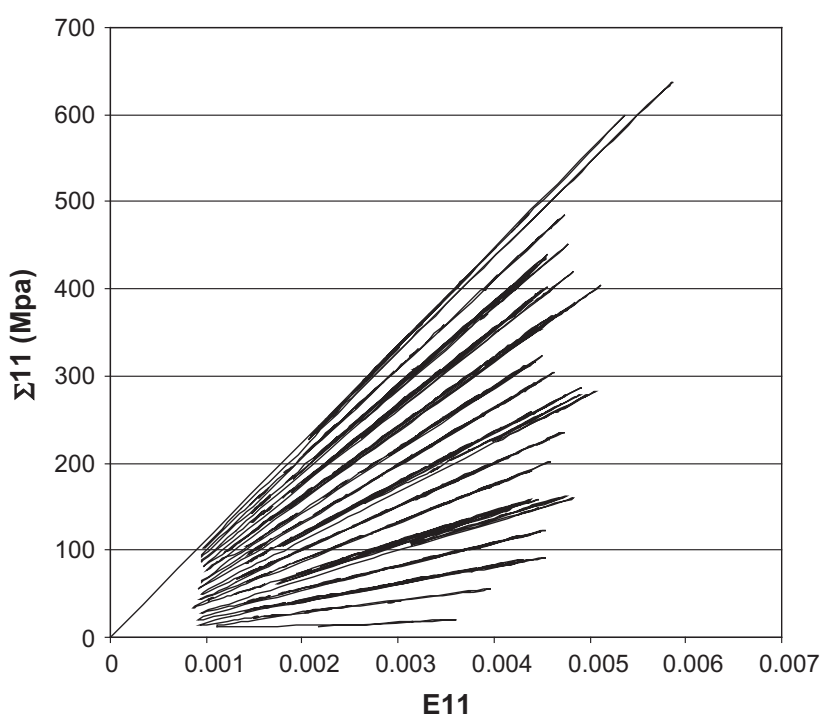

(a)

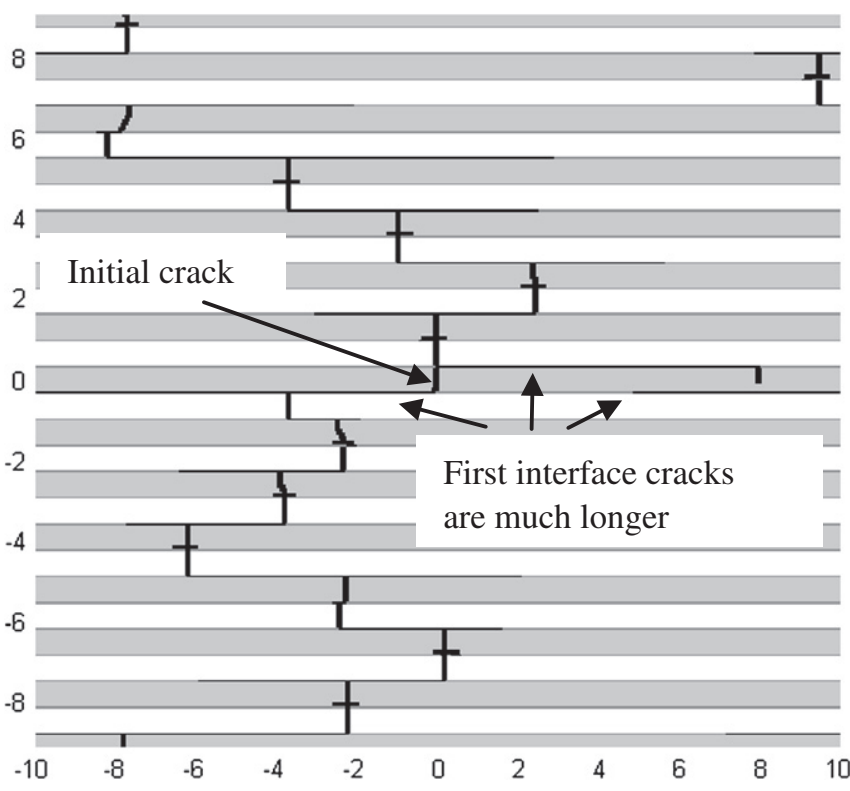

(b)

Fig. 5. Global response (a) and fracture pattern (b) of the basic cell under biaxial tension.

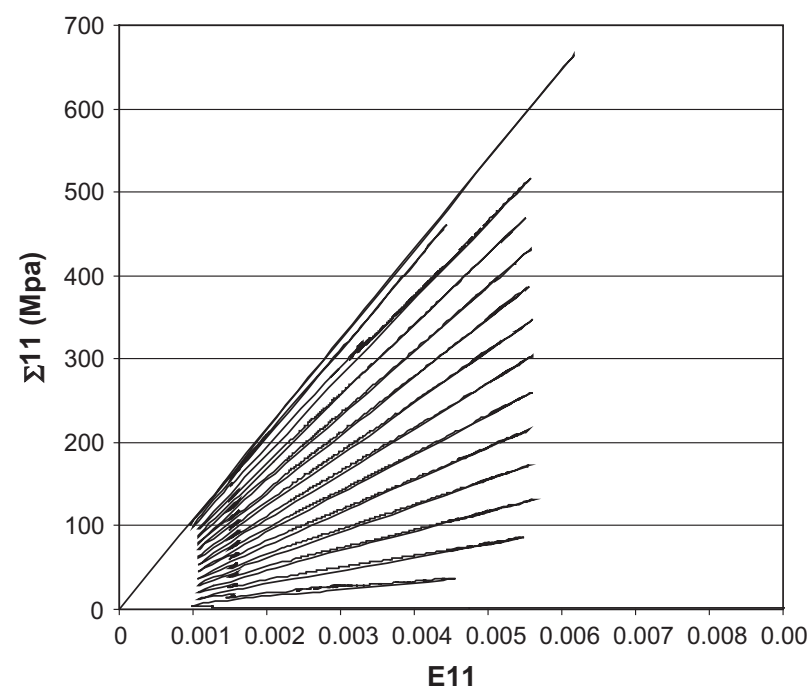

(a)

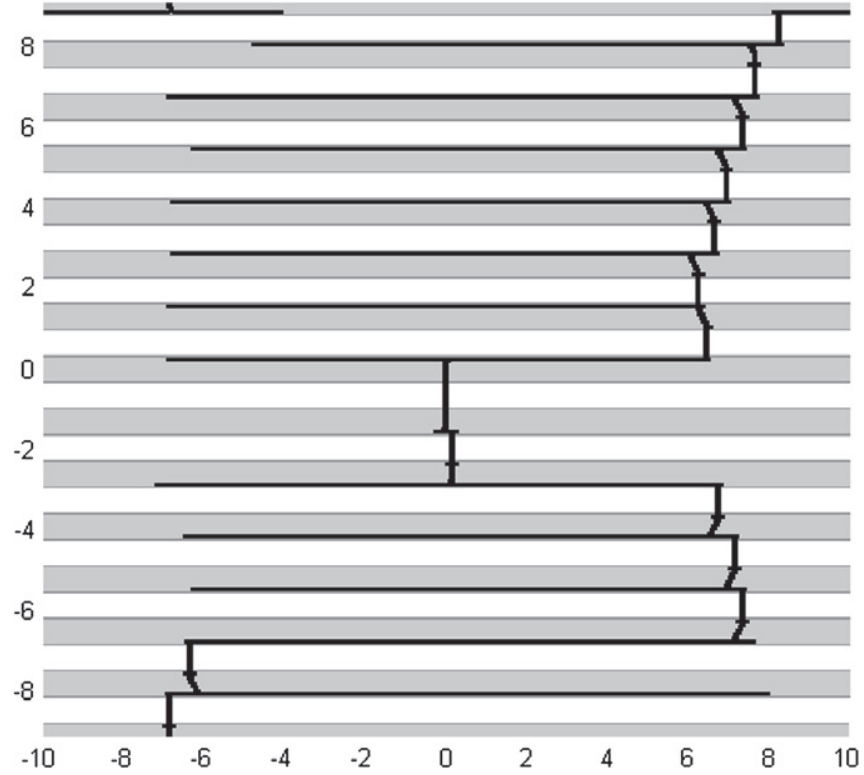

(b)

Fig. 6. Global response (a) and fracture pattern (b) of the basic cell with strong interfaces.

In the following, we will examine the influence of different material and loading parameters on the fracture of the composite.

We present hereafter a FFT simulation with a reference configuration, i.e., the basic cell subjected to a uniaxial tension by imposing average stresses $\Sigma_{11}>0$ and $\Sigma_{22}=\Sigma_{12}=0$ as remote loads.

\subsubsection{Basic cell subjected to uniaxial tension}

We first present a FFT simulation with a reference configuration, i.e., the basic cell subjected to a uniaxial tension by imposing average stresses $\Sigma_{11}>0$ and $\Sigma_{22}=\Sigma_{12}=0$ as remote loads. A weak interphase was chosen with $\sigma_{c}=40 \mathrm{MPa}$ and $K_{I c}=20 \mathrm{MPa} \sqrt{\mathrm{mm}}$.
Fig. 4a illustrates the global response, i.e., the $E_{11}-\Sigma_{11}$ curve of the cell during the loading. Fig. $4 \mathrm{~b}-\mathrm{d}$ shows the fracture patterns of the cell at the beginning, the middle and the end of the failure process. With the aid of these figures, we can describe the fracture process of the composite as follows:

- Under uniaxial tension, the global response of the composite laminate presents a saw-tooth snap-back feature. Each tooth represents the crack growth though a fibre layer.

- When a short crack grows in a layer of matrix and meets a fibre, a high level load is needed to overcome this energy barrier. It 
first deviates a little into the interface or in the matrix before entering in the fibre.

- When the crack grows in a layer of fibre and meets the matrix, it deviates slightly in the matrix before penetrating into the matrix. As the matrix strength is much lower than that of the fibre, the remote load drops significantly.

- As the transversal main crack grows and becomes longer, the length of its deviations into the interfaces increases when it meets a fibre.

- We can remark that the interface debonding acts as a crack arrestor as described in previous studies [3]. When the crack propagates in an interface, the crack tip mode mixity varies from mode I toward mode II. Its growth requires more and more energy according to the interface fracture criterion (Eq. (15)).

- As a result of the interface debonding, the final fracture surfaces take a stair form as observed in previous experimental studies $[40,41]$.

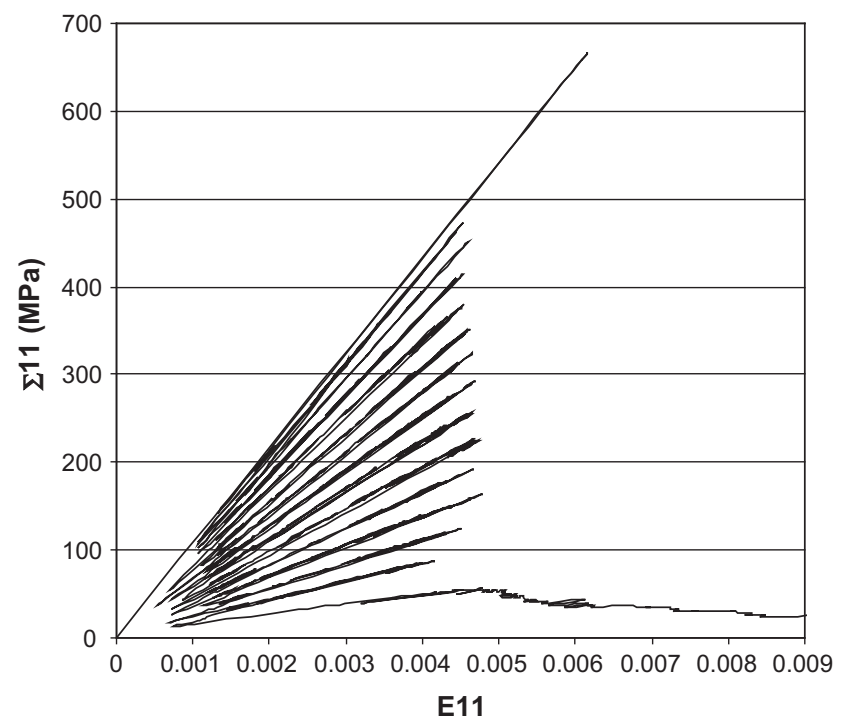

(a)

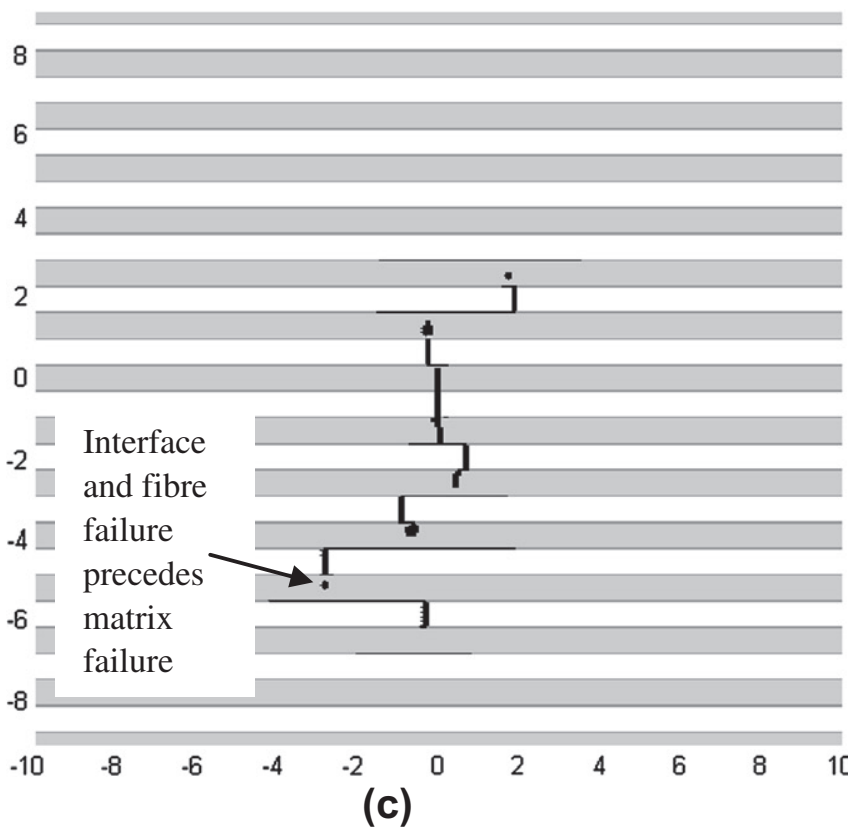

\subsubsection{Case of the biaxial tension}

In the second calculation, the same basic cell is subjected to the average strains $E_{11}>0$ and $E_{22}=E_{12}=0$. The condition $E_{22}=0$ engenders a transversal tension and therefore changes the fracture feature of the composite.

In Fig. 5a, we plot the average strain-stress curve of the cell during the loading. Fig. 5b illustrates the crack patterns of the cell at the end of the failure.

We can remark that even though the global response seems to only be slightly affected by the load change, the fracture patterns are quite different from that under uniaxial loading. Contrary to the case of uniaxial loading, the first interface cracks (Fig. 5b) under biaxial tension are very long. The subsequent interface cracks become shorter as the biaxial tension decreases but still quite long (several millimetres). Consequently, the degradation of the transversal stiffness is more important compared to the case of uniaxial loads.

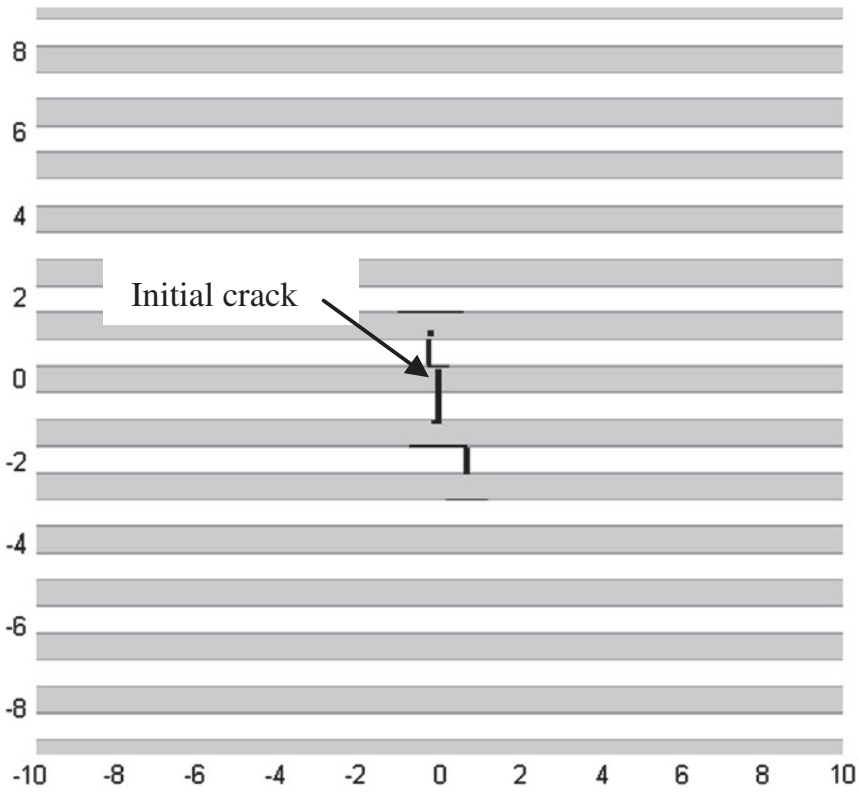

(b)

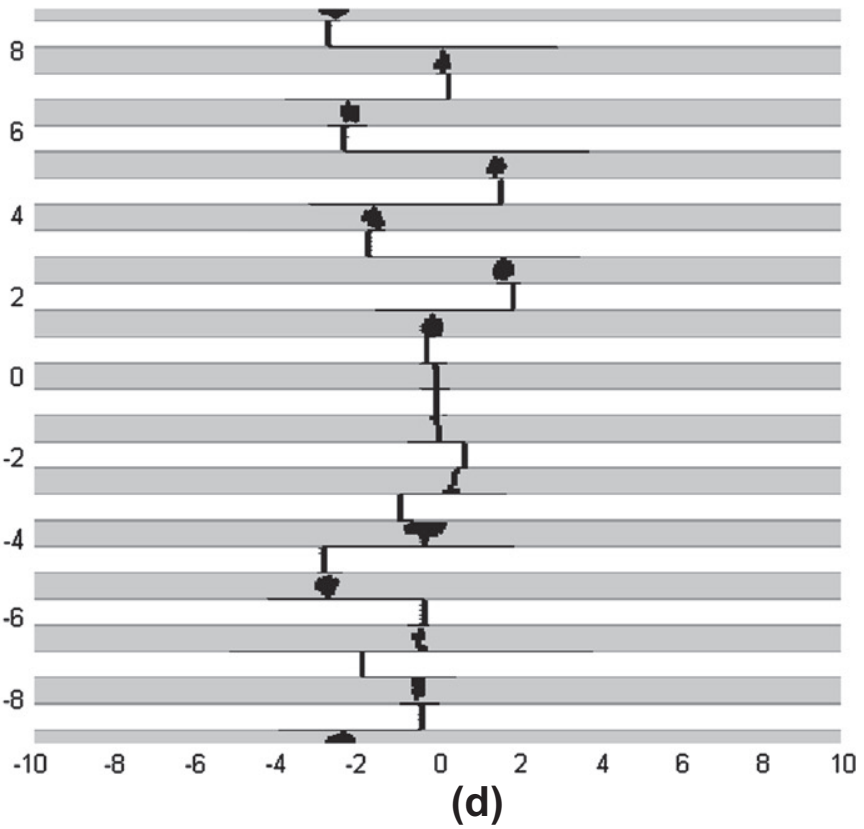

Fig. 7. Global response (a) and fracture patterns at the beginning (b), the middle (c) and the end (d) of the failure process of the basic cell with tough matrix. 


\subsubsection{Case of strong interfaces}

If the strength and the toughness of the interphase are larger than those in the above-presented simulations, for example, $\sigma_{c}=60 \mathrm{MPa}$ and $K_{I c}=30 \mathrm{MPa} \sqrt{\mathrm{mm}}$, different numerical results can be obtained. In this case, the strength and the toughness of the interphase are larger than the half of the corresponding values of the matrix. In Fig. 6a, we show the global response of the cell during the loading. Fig. $6 \mathrm{~b}$ illustrates the cracking patterns at the end of the fracture. From these figures, we can remark that first, the remote stress required to break a layer of fibre is higher comparing to that with a weaker interface, and consequently the fracture of this composite is more energy consuming. Second, as the interfaces are stronger, the crack does not deviate into the interface before it passes across a fibre, but continues to propagate in the matrix nearby and by following a parallel path to the interface. Comparing to the composite with weaker interfaces, the sub-interface crack is even longer. This is because that even though the matrix is stronger than the interphases, its fracture follows a non-local maximal principal stress criterion but not a mode-dependent criterion as that defined for an interface. Consequently, there is no such a mode-resistance behaviour for the sub-interfacial cracking.

\subsubsection{Case of large matrix toughness}

In practice, epoxies with large fracture toughness are often chosen in order to improve the global toughness of the composite. These epoxies may manifest plasticity or viscosity that should be taken into account in the modelling. In the following calculation, the same basic cell subjected to a uniaxial tension with a tougher matrix was simulated, namely $K_{I c}=200 \mathrm{MPa} \sqrt{\mathrm{mm}}$ while the ultimate stress remains unchanged. In this case, the non-local action radius of the matrix becomes much larger with $R=1.486 \mathrm{~mm}$.

Fig. 7a illustrates the $E_{11}-\Sigma_{11}$ curve of the cell during the loading. Fig. $7 b-d$ shows the fracture patterns of the cell at the beginning, the middle and the end of the failure process. The global response of the cell does not manifest significant difference from that with a brittle matrix apart from the residual strength after the failure of all the fibres. As the fibres are always much stronger and tougher than the matrix, their failure is still predominate. The visible quasi-brittle behaviour is due the larger toughness of the matrix. From Fig. 7b-d, we can observe the sequential and spatial details of the cell failure. Compared with the cell fracture with a weak matrix (Fig. 4), following observations can be made:

- The failure of the fibres and interfaces always precedes the failure of their adjacent matrix as the matrix becomes much tougher.

- The damaged zones in the matrix become larger.

- Since the interface crack initiates before the main crack touches the interface, the opening of the interface crack decreases and as a consequence, the interface cracks are shorter.

The numerical simulations presented in this section show the validity and the efficiency of the proposed failure model. Its capacity in describing different fracture mechanisms of the composite materials allows for detailed fracture prediction of this kind of composites.

\section{Concluding remarks}

In this work, we have established a non-local fracture model for composite laminates and resolved it by using the Fast Fourier Transforms (FFT). An attempt was made to model some of the important failure mechanisms in composite laminates, including the crack growth in matrix and fibres, especially the mixed-mode interfacial cracking and its role in the fracture of the whole struc- ture. For crack propagation in the matrix and the fibres, the maximal stress criterion was adapted by using the non-local concept. This non-local criterion is equivalent to the Griffith-Irwin criterion when a macrocrack is formed and to the maximal stress criterion in the case of a regular stress field. Consequently, the proposed fracture model is capable to predict crack initiation as well as crack growth. For matrix/fibre interfacial debonding, the crack growth criterion developed in Li [3] was adapted and transformed to a non-local strength criterion. The proposed model is able to describe the interface fracture mechanism observed in many experiments, according to which the interfacial fracture toughness increases with the increase of mode-II/mode-I ratio. Another particularity of the proposed approach is its ability to deal with an interface by a thin layer of interphase. The interface fracture is therefore considered exactly as that of a bulk material and no special interface separating laws is required as those defined in the cohesive models. As a consequence, the present model provides the same performance as the cohesive models by avoiding the associated convergence difficulties.

The FFT-based formulation has the advantage of leading to accurate numerical results for a periodic structure. Its possibility to profit from high resolution in discretization allows for a detailed description of the microstructure in heterogeneous materials. Consequently, this approach is particularly interesting for fracture simulations of composites in different scales. Moreover, the proposed approach enables an automatic selection of crack growth without calculating the toughness of each crack tip. Consequently, multiple crack growth problems can easily be dealt with. We believe that further studies in this direction will allow for more efficient damage models and provide abundant information on fracture process in linear or non-linear composite materials, especially in the microscopic scale.

\section{References}

[1] Moulinec H, Suquet P. A fast numerical method for computing the linear and the nonlinear mechanical properties of composites. CR Acad Sci Paris Ser II 1994;318:1417-23.

[2] Moulinec H, Suquet P. A numerical method for computing the overall response of nonlinear composites with complex microstructure. Comput Methods Appl Mech Eng 1998;157:69-94.

[3] Li J. Debonding of the interface as 'crack arrestor'. Int J Fract 2000;105:57-79.

[4] Lemaitre J, Chaboche JL. Mechanics of solid materials. Cambridge: Cambridge University Press; 1990.

[5] Basu S, Waas LM, Ambur RD. Prediction of progressive failure in multidirectional composite laminated panels. Int $\mathrm{J}$ Solids Struct 2007;44(9):648-2676.

[6] Maimi P, Camanho PP, Mayugo JA. A continuum damage model for composite laminates: Part I - constitutive model. Mech Mater 2007;39(10):897-908.

[7] Matzenmiller A, Lubliner J, Toylao RA. A constitutive model for anisotropic damage in fibre-composites. Mech Mater 1995;20(2):125-52.

[8] Chow CL, Yang F. Three-dimensional inelastic stress analysis of carbon fibre/ epoxy composite laminate using continuum damage mechanics. Int J Damage Mech 1997;6(1):23-50.

[9] Pineda EJ, Waas AM, Bednarcyk BA, Collier CS, Yarington PW. Progressive damage and failure modeling in notched laminated _ber reinforced composites. Int J Fract 2009;158:125-43.

[10] Lin WP, Hu HT. Nonlinear analysis of fibre-reinforcrd composite laminates subjected to uniaxial tensile load. J Compos Mater 2002;36(12):429-50.

[11] Boutaous A, Peseux B, Cornet L, Bélaidi A. A new modeling of plasticity coupled with the damage and identification for carbon fibre composite laminates. Compos Struct 2006;74(1):1-9.

[12] Barbero EJ. An inelastic damage model for fibre reinforced laminates. J Compos Mater 2002;36(8):941-62.

[13] Hashin Z. Failure criteria for unidirectional fiber composites. J Appl Mech 1980;47:329-34.

[14] Hoffman O. The brittle strength of orthotropic materials. J Compos Mater 1967;1(2):200-6.

[15] Yamada SE, Sun CT. Analysis of laminated strength and its distribution. J Compos Mater 1978;12:275-84.

[16] Aziz VD, Tsai SW. Anisotropic strength of components. Exper Mech 1965;5:286-8.

[17] Tsai SW, Wu EM. Ageneral theory of strength for anisotropic materials. J Compos Mater 1971;5(1):58-80. 
[18] Barenblatt G. The formation of equilibrium cracks during brittle fracture. J Appl Math Mech 1959;23:434-44.

[19] Dugdale D. Yielding of steel sheets containing slits. J Mech Phys Solids 1960;8:100-4.

[20] Hutchinson JW, Suo Z. Mixed mode cracking in layered materials. Adv Appl Mech 1992;29:63-191.

[21] Sun CT, Jin ZH. Modeling of composite fracture using cohesive zone and bridging models. Compos Sci Technol 2006;66(10):1297-302.

[22] Shin CS, Wang CM. An improved cohesive zone model for residual notched composites. Int J Fract 2004;133(2):107-37.

[23] Lapczyk I, Hurtado JA. Progressive damage modelling in fiber-reinforced materials. Composites Part A 2007;38(1):2333-41.

[24] Rami HA. Cohesive micromechanics: a new approach for progressive damage modelling in laminated composites. Int J Damage Mech 2009; 18(8):691-719.

[25] De Xie, Waas AM. Discrete cohesive zone model for mixed-mode fracture using finite element analysis. Eng Fract Mech 2006;73(13):1783-96.

[26] Michel JC, Moulinec H, Suquet PA. Computational method based on augmented Lagrangians and fast Fourier transforms for composites with high contrast. Comput Modell Eng Sci 2000;1(2):79-88.

[27] Pijaudier-Cabot G, Bazant ZP. Nonlocal damage theory. J Eng Mech ASCE 1987;113:1512-33.

[28] Li J, Tian XX, Abdelmoula R. Fracture in brittle and quasi-brittle materials simulated by FFT method. Int J Fract, submitted for publication.

[29] Williams ML. The bending stress distribution at the base of a stationary crack. J Appl Mech 1961;28:78-82.
[30] Griffith AA. The phenomena of rupture and flow in solids. Philos Trans Roy Soc London 1920;221:163-98.

[31] Irwin G. Linear fracture mechanics, fracture transition and fracture control. Eng Fract Mech 1968;1:241-57.

[32] Leguillon D. Strength or toughness? A criterion for crack onset at a notch. Eur JMech A/Solids 2002;21:61-72.

[33] Li J, Zhang XB. A criterion study for non-singular stress concentrations in brittle or quasi-brittle materials. Eng Fract Mech 2006;74:505-23.

[34] Liechti KM, Chai YS. Asymmetric shedding in interfacial fracture under inplane shear. J Appl Mech 1992;59:295-304.

[35] Cao HC, Evans AG. An experimental study of the fracture resistance of biomaterial interfaces. Mech Mater 1989;7:295-304.

[36] Freed Y, Banks-Sillis L. A new cohesive zone model for mixed mode interface fracture in bimaterials. Eng Fract Mech 2008;75:4583-93.

[37] Hutchinson JW, Mear M, Rice JR. Crack paralleling an interface between dissimilar materials. J Appl Mech 1987;54:828-32.

[38] Malyshev BM, Salganik RL. The strength of adhesive joints using the theory of crack. Int J Fract Mech 1965;1:114-28.

[39] Sih GC, Rice JR. The bending of plates of dissimilar materials with cracks. J Appl Mech 1964;31:477-82.

[40] Raynaud C. Céramique lamellaires monolithiques en carbure de silicium. PhD thesis, Ecole Nationale des Mines de St Etienne and Institut National Polytechnique de Grenoble; 2002.

[41] Tariolle S. Carbure de bore monolithique poreux et composites laminaires. PhD thesis, Ecole Nationale des Mines de St Etienne and Institut National Polytechnique de Grenoble; 2003. 